\title{
Templated Self-Assembly in Three Dimensions Using Magnetic Levitation
}

\section{Citation}

Ilievski, F., Mirica, K. A., Ellerbee, A. K. and Whitesides, G. M. 2011. "Templated Self-Assembly in Three Dimensions Using Magnetic Levitation." Soft Matter 7, no. 19: 9113-9118.

\section{Permanent link}

http://nrs.harvard.edu/urn-3:HUL.InstRepos:12967699

\section{Terms of Use}

This article was downloaded from Harvard University's DASH repository, and is made available under the terms and conditions applicable to Open Access Policy Articles, as set forth at http:// nrs.harvard.edu/urn-3:HUL.InstRepos:dash.current.terms-of-use\#OAP

\section{Share Your Story}

The Harvard community has made this article openly available.

Please share how this access benefits you. Submit a story.

\section{Accessibility}




\title{
Templated Self-Assembly in Three Dimensions Using Magnetic Levitation
}

\author{
Filip Ilievski ${ }^{1}$, Katherine A. Mirica ${ }^{1}$, Audrey K. Ellerbee ${ }^{1}$, and George M. Whitesides ${ }^{1,2, *}$ \\ ${ }^{1}$ Department of Chemistry and Chemical Biology, Harvard University \\ 12 Oxford Street, Cambridge, MA 02138 \\ ${ }^{2}$ Kavli Institute for Bionano Science \& Technology, \\ 29 Oxford Street, Cambrdige MA, and \\ Wyss Institute for Biologically Inspired Engineering, Harvard University \\ 3 Blackfan Circle, Boston, MA 02115
}

*Corresponding author, email: gwhitesides@gmwgroup.harvard.edu 


\begin{abstract}
Although self-assembly (SA) in two dimensions (2D) is highly developed (especially using surfaces as a templates), SA in three dimensions (3D) has been more difficult. This paper describes a strategy for SA in 3D of diamagnetic plastic objects ( $\mathrm{mm}$ - to cm-sized in this work, but in principle in sizes from $\sim 10 \mu \mathrm{m}$ to $\mathrm{m}$ ) supported in a paramagnetic fluid by a non-uniform magnetic field. The magnetic field and its gradient levitate the objects, template their selfassembly, and influence the shape of the assembled cluster. The structure of the 3D assembling objects can be further directed using hard mechanical templates - either the walls of the container or co-levitating components - which coincide spatially with the soft template of the magnetic field gradient. Mechanical agitation anneals the levitating clusters; the addition of photocurable adhesive, followed by UV illumination, can permanently fuse components together.
\end{abstract}


The problem of how best to use self-assembly (SA) to organize meso-scale components (from sizes less than $\mathrm{mm}$ to greater than $\mathrm{cm}$ ) into three-dimensional (3D) assemblies is unsolved: structures tend to be dominated by gravitational forces, rather than by interaction between the components. In fact, there are no general strategies for assembly in 3D, other than those involving mechanical processes, such as the use of machines ${ }^{1}$ directed or programmed by humans. Self-assembly in 3D at the molecular scale is, of course, ubiquitous, but these processes operate under different constraints than those for larger objects: the molecular forces experienced by thermal collisions - Brownian motion — are larger than forces due to gravity, and molecules remain suspended in solution indefinitely.

In the laboratory, processes based on $\mathrm{SA}^{2}$, are most successful, and highly developed, at surfaces (i.e., in 2D). Examples at different scales include the assembly of ordered monolayers of alkanethiolates (SAMs) on gold ${ }^{3}$, of colloid particles into crystals and photonic band-gap structures $^{4}$, of bubbles into crystalline bubble rafts ${ }^{5}$, of microspheres into ordered arrays ${ }^{6}$ and of chips onto credit cards ${ }^{7}$. The presence of a templating surface both simplifies and limits SA. In general, the most successful laboratory demonstrations of self-assembly use a single kind of a simple component (e.g., uniform spheres); crystals of spheres that have been explored actively for use in photonics ${ }^{8}$, optics $^{8}$, and electronics ${ }^{9}$ provide examples. There are, of course many elegant examples of self-assembled molecules and molecular aggregates (for example, molecular crystals, liquid crystals ${ }^{10}$, phase-separated block copolymers ${ }^{11}$, proteins $^{12}$, and protein segregates ${ }^{12}$ ). The processes that generate these structures are, however, not easily subject to design or adapted to non-molecular components, and the most "elementary" of them (e.g., crystallization of simple organic molecules from non-polar solvents) still seem intractably difficult to model or simulate. 
The objective of this work is to explore the potential of an unfamiliar approach to 3D self-assembly: magnetic levitation (MagLev). In MagLev, the gravitational forces that make 3D SA so difficult to accomplish using mesoscopic components in a gravitational field are reduced or cancelled using magnetic forces.

Magnetic levitation (MagLev) has previously been used to separate materials based on differences in density ${ }^{13-22}$ and for measuring density, but the only examples in the literature pointed toward SA have produced only disordered aggregates ${ }^{23-27}$. This paper expands on a strategy we have recently described ${ }^{28}$ that makes it possible to suspend objects in $3 \mathrm{D}$, without requiring contact with solids, and ordered according to density and the relative influence of magnetic and gravitational forces.

MagLev has eight characteristics that make it particularly attractive as a strategy for 3D SA of mm-scale objects. i) It is not limited to surfaces, and avoids some of the limitations imposed by gravity in conventional SA. ii) SA based on MagLev can be made reversible — a necessary requirement for minimizing the density of defects in the assembled clusters ${ }^{29-31}$. iii) MagLev SA can use components from a wide range of materials. Using the simple, relatively low-field magnets we employ here, objects with densities between $1-3 \mathrm{~g} / \mathrm{cm}^{3}$ can easily be manipulated; this range includes most organic polymers. iv) MagLev is applicable to soft, fragile, and sticky objects; such objects are otherwise very difficult to handle. v) Composite objects can be assembled from several different materials. vi) Multiple components with different sizes, shapes or properties can self-assemble into asymmetrical, ordered structures using MagLev. vii) MagLev can be adapted to a wide range of problems in SA because it is influenced by a number of independently controllable parameters (the densities and shape of the objects, the density and magnetic susceptibility of the paramagnetic liquid, the shape of the 
magnetic field, the shape of the container, and other readily controlled parameters such as temperature and pressure). viii) MagLev induces SA over convenient time-scales (the time it takes to form well-ordered structures using mm-scale objects MagLev is on the order of seconds to minutes). The ability to anneal the self-assembled structures by mechanical agitation is also useful.

This paper describes the 3D SA of mm-sized diamagnetic objects, suspended in a paramagnetic liquid, in a non-uniform magnetic field. Using this system, we studied the effects of soft templates (produced by the magnetic field), and hard templates (both levitating objects and the walls of the container), on the packing and shaping of magnetically levitating clusters of both spherical and non-spherical objects. The device we used for MagLev consists of two inexpensive $\mathrm{NdFeB}$ permanent magnets oriented with like poles facing each other (antiHelmholtz configuration), and a container containing a paramagnetic medium (an aqueous solution of $\mathrm{Mn}^{+2}$ ). Objects suspended in the paramagnetic medium and positioned between the two magnets assemble and orient spontaneously. The final position of the objects in the liquid, and their self-assembly, is directed by a competition between gravitational forces, magnetic forces, and steric interactions (mechanical forces from physical contact) among and between the objects and the container.

Experimental Design. The repulsive force exerted by non-uniform magnetic fields on diamagnetic materials is typically negligible for most materials, and is insufficient to suspend them against gravity in air using permanent magnets (bismuth and graphite are exceptions, but the strength of their repulsion from a region of high field is still small compared to the strength of attraction of most paramagnetic objects). A simple strategy for achieving MagLev of diamagnetic objects with permanent magnets is to suspend these objects in a paramagnetic fluid, 
and to place that fluid in a magnetic field gradient generated using two magnets oriented with like poles facing each other. In this arrangement, the paramagnetic medium is attracted towards the regions of high magnetic field, and displaces the diamagnetic object towards regions of lower magnetic field; this exchange of paramagnetic matter for diamagnetic in regions of high magnetic field enables magnetic levitation.

The levitating objects come to rest at the location where the gravitational $\left(\dot{F}_{g}\right)$ and magnetic $\left(\dot{F}_{\text {mag }}\right)$ forces balance (Eq. 1). In this equation, $\rho_{c}$ and $\rho_{m}$ are the density of the levitating component and the paramagnetic medium, respectively $\left[\mathrm{kg} / \mathrm{m}^{3}\right], \chi_{s}$ and $\chi_{m}$ are the magnetic susceptibilities of the sample and the medium [unitless in SI], $B$ is the magnitude of the magnetic field $[\mathrm{A} / \mathrm{m}], V$ is the volume of the sample $\left[\mathrm{m}^{3}\right], \mu_{o}$ is the permeability of free space $[=$ $\left.1.26 \times 10^{-6} \mathrm{~m} \mathrm{~kg} \mathrm{~s}^{-2} \mathrm{~A}^{-2}\right]$ and $g$ is the gravitational constant $\left[=9.81 \mathrm{~m} \mathrm{~s}^{-2}\right]$.

$$
\stackrel{\mathbf{r}}{F_{g}}+{\stackrel{\mathbf{r}}{F_{m a g}}}_{\text {ma }}=\left(\rho_{c}-\rho_{m}\right) V V_{g}^{\mathbf{r}}+\frac{\left(\chi_{m}-\chi_{c}\right)}{\mu_{0}} V(\stackrel{\mathbf{r}}{B} \cdot \stackrel{\mathbf{r}}{\nabla}) \stackrel{\mathbf{r}}{B}=0
$$

The NdFeB magnets we used here are inexpensive ( $\sim 20$ when purchased individually and significantly less in bulk), and generate large magnetic fields (remanent field, $M_{R}=1.1$ $\mathrm{kA} / \mathrm{m}$ and field at the magnet surface $\sim 0.4 \mathrm{~T})$. Their large coercivity $\left(H_{C}=1.1 \mathrm{~T}\right)$ makes them resistant to demagnetization when multiple magnets are used in an anti-Helmholtz arrangement. Two rectangular prism-shaped magnets in an anti-Helmholtz configuration generate a region of low magnetic field between the magnets — an oblate-spheroid-shaped "magnetic bottle"; this configuration is especially useful for 3D SA for three reasons. i) The system centers and aligns levitating diamagnetic objects or clusters along a vertical centerline between the magnets (dotted line in Figure 1a), because the magnitude of the magnetic field is minimal in that region of the $x$ $y$ plane. ii) It levitates objects that are either more dense or less dense than the paramagnetic 
solution: objects that are more dense than the medium sink in the absence of the magnetic field, and their levitation is enabled by the bottom magnet; objects that are less dense than the medium float in the absence of an applied magnetic field, and their levitation (or "reverse levitation") is enabled by the top magnet. iii) The magnetic field gradient can be easily altered by changing the distance between magnets.

The paramagnetic solution enables levitation in two ways: i) it provides a buoyant force that counteracts gravity, and ii) it controls the magnitude of the magnetic force experienced by the paramagnetic medium, and thus contributes another force to those experienced by the diamagnetic objects ${ }^{13}$. We used 1.0-1.5 $\mathrm{M} \mathrm{MnCl}_{2}\left(\chi_{m}=7 \times 10^{-4}\right)$ solutions in water $(\rho=1.10-$ $\left.1.15 \mathrm{~g} / \mathrm{cm}^{3}\right)$ for levitation, and we added non-ionic surfactant $(0.1 \% \mathrm{v} / \mathrm{v}$ Triton X-100 or Tween20) to reduce friction and hydrophobic interactions between objects and thus to reduce defects in assembly. Solutions of $\mathrm{MnCl}_{2}$ in water have high magnetic susceptibility $\left(\chi_{m} \approx 10^{-3}\right.$ for solutions $\approx 4 \mathrm{M}$ and decreases linearly with concentration), and low cost $(<\$ 0.05 / \mathrm{g}$ for quantities used in a lab experiment, and significantly less in bulk). They enable levitation of objects that range in density from $1-2 \mathrm{~g} / \mathrm{cm}^{3}$ (and up to $3 \mathrm{~g} / \mathrm{cm}^{3}$ with $\mathrm{MnBr}_{2}$ and/or addition of other diamagnetic co-solutes, such as $\mathrm{CaCl}_{2}$ or $\mathrm{ZnCl}_{2}$; the added $\mathrm{Ca}^{+2}$ or $\mathrm{Zn}^{+2}$ diamagnetic ions do not significantly change the susceptibility, but the dissolved salt changes the density of the solution). This range of densities is well-suited for levitating most organic polymers.

In most of these studies we used spherical objects as components. Spherical objects are well-suited for initial experiments because surface interactions between two spheres are minimal; strong surface interactions may inhibit sliding of objects towards the equilibrium structures, and can lead to the formation of kinetically trapped, metastable structures. 
We selected polymethylmethacrylate (PMMA, density $=1.19 \mathrm{~g} / \mathrm{cm}^{3}$ ) for most of the diamagnetic components; this polymer is homogeneous, inexpensive, commercially available in many shapes and sizes, and easily machined. Laser cutting generated non-spherical objects from sheets of PMMA.

The assembly of multiple components into ordered, equilibrium structures usually requires agitation. We annealed the less stable aggregates formed initially into more stable structures by mechanically agitating the container (various prism-shaped boxes, $2-8 \mathrm{~cm}$ in length), using an attached, unbalanced micro-motor (the kind that produces vibrations in a cell phone). This rotor produces vibration at $250 \mathrm{~Hz}$; the vibrations are transmitted through the liquid and agitate the cluster.

Self-Assembly of Spheres in the Absence of Rigid Templates. The magnetic field gradient creates a "magnetic bottle" that traps levitating objects; the shape of this bottle (or "magnetic template") determines how multiple levitating objects pack within it. Figure $1 \mathrm{~b}$ illustrates the self-assembly of clusters of spheres within a magnetic template produced by the MagLev device. We compare non-agitated clusters to those annealed with mechanical agitation. The left column of Figure $1 \mathrm{~b}$ shows examples of clusters of spheres that formed spontaneously after placing the spheres in the container filled with the paramagnetic medium, allowing them to sink, and positioning the container between the magnets within the MagLev device. Horizontal sheets formed without agitation for clusters consisting of up to seven spheres (Figure 1b, left column) for all sphere sizes we used in our experiments ( $0.06 \mathrm{in}-0.25 \mathrm{in} / 1.6 \mathrm{~mm}-6.4 \mathrm{~mm}$ in diameter). In this case, the magnetic field essentially provides a very shallow bowl with a slight magnetic "rim" around the region where the spheres are levitating, and permits the formation of 
the flat horizontal sheets shown in Figure 1b. Agitation, either manually (by tapping) or by vibrating the container promotes the formation of ordered clusters (Figure 1b, right column).

Sheets comprising eight or more spheres experience lateral magnetic forces from the "rim" that push spheres $(1.6 \mathrm{~mm}-6.4 \mathrm{~mm}$ in diameter $)$ at the margins toward the center and prevent the expansion of the ordered sheets upon addition of spheres; these clusters remain disordered even after 10 minutes of mechanical agitation. To increase the size of a stable flat cluster, we increased the magnet-to-magnet distance to $70 \mathrm{~mm}$, and thereby extended the template laterally and flattened it (the local curvature around the centerline was reduced; compare the COMSOL simulations of the magnetic field shape in Figure 2). In this configuration, planar close-packed sheets of up to nine spheres $(0.06$ in -0.25 in / $1.6 \mathrm{~mm}-6.4$ $\mathrm{mm}$ in diameter) form.

Freely-levitating clusters comprising ten or more spheres $(1.6 \mathrm{~mm}-6.4 \mathrm{~mm}$ in diameter $)$ did not form a well-ordered cluster when mechanically agitated in this MagLev device, even at the larger magnet separation; the cluster, however, levitated such that its center of mass remained at constant height, and additional spheres increased the thickness and lateral extent of the cluster (Figure 2).

Self-Assembly of Spheres in the Presence of Rigid Templates. Templates that promote ordered structures by self assembly need to have well-defined edges (either physical or energetic); the magnetic gradient is smooth and lacks such features, and may not promote the formation of multilayer crystalline clusters with well-defined edges. The formation of wellordered clusters of more than seven spheres thus required the use of a rigid physical template (or the use of larger magnets at a larger separations); we used physical templates to order larger clusters and to induce alternate (non-close-packed) ordering. Solid objects act as templates for 
the SA of levitating spheres by providing a surface along which the spheres can pack. Contact between a flat surface and aggregates of spheres leads to the hexagonal packing of those spheres in direct contact with the template; agitating these levitating objects extends this order through the cluster (including to those spheres not in contact with the template, see Figure S 1 ). We demonstrate two types of templates: i) the flat walls of a container, and ii) co-levitating objects.

Any rigid flat surface that comes in contact with the cluster will act as a template and can induce ordering in the cluster. For example, bringing a cluster of 60 spheres into contact with the wall of the container, with agitation, promotes the formation of a face-center cubic packed multilayer cluster (Figure S 1). Packing of levitating spheres in narrow containers follows rules outlined previously ${ }^{32-34}$, and is discussed in the Supplemental Materials.

Addition of rigid objects that levitate in contact with the cluster induces ordering in otherwise disordered clusters (Figure 3a), and, by properly designing the levitating template, we are able to form different lattices and structures. We demonstrate two types of templates: i) 'enveloping templates' induce order by wrapping around the cluster; ii) 'displacing templates,' displace spheres occupying the lowest regions of magnetic field within the magnetic trap. For example, co-levitating flat plates induce crystallization in aggregates of 70 spheres (Figure 3a). A co-levitating rectangular frame with the same density as the spheres templated the square packing of spheres (Figure 3b). The surfaces and edges of levitating objects can also induce ordered packing of other objects around them (Figure 3c). To generate the structures shown in Figure 3, we simply placed both the spheres and the templates simultaneously into a container filled with a paramagnetic medium, and positioned the container in the magnetic field. 
Levitating templates provide a degree of control and freedom in designing selfassembling structures not available to other methods of SA. Enveloping and displacing templates can be co-levitated simultaneously to create complex multi-component structures.

Self-Assembly of Complex Structures from Components with Different Densities. By using components with different average densities, we can control the vertical placement of the levitating objects with respect to one another. This control enables the self-assembly of more complex structures by a process analogous to a layer-by-layer assembly. Figure 4a illustrates multiple levitating objects with different densities that have self-assembled into layered structures according to their density. In the absence of additional mechanical templates that restrict the positions of components, the shape of the magnetic template controls the structure of each layer. The introduction of a physical template, in the form of a wall or co-levitating object, induces order in the separated clusters (Figure S 1, Figure S 2). By patterning the density distribution of each object (in addition to the average density), we can also control the orientation and placement of the individual objects in the final, self-assembled structure (see video S1 in online supplemental materials). To achieve these structures, no special layering of the objects was necessary - all objects were placed in the container at the same time, and, upon agitation, separated at different heights according to their average density.

Figure 5 demonstrates several uses of this method to program the assembly of complex structures. We prepared several complex objects by gluing together spheres of different densities to sheets of acrylic before placing them in containers of paramagnetic solution. The objects selfassembled into layered structures directed by MagLev. Reducing the separation between magnets makes it possible to bring separate layers into contact. 


\section{Joining Components in Clusters of Self-Assembled Structures. Practical applications}

usually require that structures be stable once self-assembled. To fix the SA structures we describe here permanently, we applied a photocurable adhesive after self-assembly was complete. A portion of the paramagnetic solution (typically one-sixth to one-fifth of the volume) was removed and an emulsion containing photocurable adhesive (1:100 v/v of Norland Optical Adhesive 72 in the $\mathrm{MnCl}_{2}$ solution used for levitating) was added slowly (over the course of 2 minutes) so as not to disturb the SA cluster. We permitted the adhesive to coat the cluster for five minutes, and then exposed the solution to UV light to crosslink it. The objects were then removed and rinsed (Figure 6).

Conclusion. This paper demonstrates the use of MagLev to guide the self-assembly of components in 3D. A stationary magnetic field suspends objects against gravity, and the shape of the magnetic field gradient guides them into contact with one another, and determines the initial shape of the levitating clusters. To induce order in the levitating cluster, or to influence the shape of the cluster (i.e., to make non-close-packed based cluster), we positioned surfaces of rigid, mechanical templates (e.g., walls of a container or co-levitating objects) in contact with the cluster. Tailoring the size and shape of the rigid templates promotes the formation of alternate packing of spheres to the typical hexagonal-packed structures. Manipulating the density of the objects easily controls the vertical position of levitating objects in MagLev; introducing patterns of density into individual components controls their orientation. The simultaneous levitation of multiple components with different densities enables the assembly of multilayered structures, and freely-levitating clusters can be permanently joined by the addition of photocurable adhesive in the paramagnetic solution and subsequently removed from the container. 
MagLev has several useful features as a method for 3D SA: i) it is compatible with a wide range of materials (plastics, liquids, gels, pastes); ii) it is conveniently rapid (the time it takes to form well-ordered structures using mm-scale objects MagLev is on the order of seconds to minutes); iii) it is applicable to a wide range of shapes and sizes of objects; iv) it generates assemblies reversibly: these assemblies can be disassembled by the removal of the magnetic field. Devices for MagLev can be easily modified to expand the range of structures and objects that can be self-assembled. Devices that generate higher fields and field gradients $\left(\dot{B}^{\prime} \cdot \dot{\nabla}\right) \dot{B}^{\prime}$ can be used to self-assemble objects that have a higher density than what we explore here, or that have smaller sizes than demonstrated here. To generate differently shaped magnetic holes, the magnetic fields and magnetic field gradients can be shaped by i) use of permanent magnets with different shapes, ii) soft ferromagnetic-field-concentrators, iii) addition of electromagnets, or series of electromagnets.

MagLev also has a number of limitations: i) for our device, Brownian motion impedes the assembly of objects with diameters less than $5 \mu \mathrm{m}$, a shortcoming that can be addressed by using a device with a larger magnetic gradient; ii) levitation of large objects is limited by the sizes of the magnets and container; this limit could be, in principle, overcome by using large magnets.

MagLev is a flexible and versatile new technique for 3D SA. The freedom to program the SA of multiple complex objects should lead to improved processes for assembly of small objects, and provide an alternative to processes currently addressed by serial pick-and-place assembly by humans or robots. 


\section{Acknowledgments}

This work was supported by DARPA under award W911NF-08-1-0143.

\section{Author Contributions}

F.I., K.A.M., and G.M.W. conceived the experiments. F.I. and K.A.M. performed the experiments, and analyzed the data. A.K.E. performed simulations. All authors wrote the paper.

\section{References}

1. Quaid, A. \& Hollis, R. Cooperative 2-DOF robots for precision assembly. Proc. IEEE Int. Conf. Rob. Autom. 2188-2193 (1996).

2. Whitesides, G.M. \& Grzybowski, B. Self-assembly at all scales. Science 295, 2418-2421 (2002).

3. Bain, C.D. \& Whitesides, G.M. Molecular-level control over surface order in self-assembled monolayer films of thiols on gold. Science 240, 62-63 (1988).

4. Vlasov, Y., Bo, X., Sturm, J. \& Norris, D. On-chip natural assembly of silicon photonic bandgap crystals. Nature 414, 289-293 (2001).

5. Simpson, A.W. \& Hodkinson, P.H. Bubble raft model for an amorphous alloy. Nature 237, 320-322 (1972).

6. Holgado, M. et al. Electrophoretic deposition to control artificial opal growth. Langmuir 15, 4701-4704 (1999).

7. Smith, J.S., Hadley, M.A., Craig, G.S.W. \& Lowe, F. United States Patent: 6623579 Methods and apparatus for fluidic self assembly. (2003).

8. Joannopoulos, J.D., Villeneuve, P.R. \& Fan, S. Photonic crystals: putting a new twist on light. Nature 386, 143-149 (1997). 
9. Hamley, I. Nanostructure fabrication using block copolymers. Nanotechnology 14, 39 (2003).

10. Kato, T. Liquid crystalline functional assemblies and their supramolecular structures. (Springer: Berlin, 2008).

11. Bates, F.S. \& Fredrickson, G.H. Block copolymer thermodynamics: theory and experiment. Annu. Rev. Phys. Chem. 41, 525-557 (1990).

12. Chayen, N.E., Helliwell, J.R. \& Snell, E.H. Macromolecular crystallization and crystal perfection. (Oxford University Press: Oxford, 2010).

13. Mirica, K.A., Shevkoplyas, S.S., Phillips, S.T., Gupta, M. \& Whitesides, G.M. Measuring Densities of Solids and Liquids Using Magnetic Levitation: Fundamentals. J. Am. Chem. Soc. 131, 10049-10058 (2009).

14. Winkleman, A. et al. Density-based diamagnetic separation: devices for detecting binding events and for collecting unlabeled diamagnetic particles in paramagnetic solutions. Anal. Chem. 79, 6542-6550 (2007).

15. Mirica, K.A., Phillips, S.T., Shevkoplyas, S.S. \& Whitesides, G.M. Using magnetic levitation to distinguish atomic-level differences in chemical composition of polymers, and to monitor chemical reactions on solid supports. J. Am. Chem. Soc. 130, 17678-17680 (2008).

16. Andres, U. Magnetohydrodynamic \& Magnetohydrostatic Methods of Mineral Separation. (John Wiley \& Sons: New York, NY, 1976).

17. Catherall, A., Lopez-Alcaraz, P., Benedict, K., King, P. \& Eaves, L. Cryogenically enhanced magneto-Archimedes levitation. New J. Phys. 7, No. 118 (2005). 
18. Catherall, A., Eaves, L., King, P. \& Booth, S. Floating gold in cryogenic oxygen. Nature 422, 579-579 (2003).

19. Ikezoe, Y. et al. Separation of feeble magnetic particles with magneto-Archimedes levitation. Energy Conv. Manag. 43, 417-425 (2002).

20. Kimura, T., Mamada, S. \& Yamato, M. Separation of solid polymers by magnetoarchimedes levitation. Chem. Lett. 1294-1295 (2000).

21. Hirota, N. et al. Magneto-Archimedes separation and its application to the separation of biological materials. Physica B 346, 267-271 (2004).

22. Mirica, K.A., Phillips, S.T., Mace, C.R. \& Whitesides, G.M. Magnetic levitation in the analysis of foods and water. J. Agr. Food Chem. 58, 6565-6569 (2010).

23. Feinstein, E. \& Prentiss, M. Three-dimensional self-assembly of structures using the pressure due to a ferrofluid in a magnetic field gradient. J. Appl. Phys. 99, 064901 (2006).

24. Takayama, T., Ikezoe, Y., Uetake, H., Hirota, N. \& Kitazawa, K. Self-organization of nonmagnetic spheres by magnetic field. Appl. Phys. Lett. 86, (2005).

25. Kimura, T., Sato, Y., Kimura, F., Iwasaka, M. \& Ueno, S. Micropatterning of cells using modulated magnetic fields. Langmuir 21, 830-832 (2005).

26. Skjeltorp, A.T. \& Helgesen, G. Condensation and ordering of colloidal spheres dispersed in a ferrofluid. Physica A 176, 37-53 (1991).

27. Islam, M.F., Lin, K.H., Lacoste, D., Lubensky, T.C. \& Yodh, A.G. Field-induced structures in miscible ferrofluid suspensions with and without latex spheres. Phys. Rev. E 67, 021402 (2003).

28. Mirica, K.A., Ilievski, F., Ellerbee, A.K., Shevkoplyas, S.S. \& Whitesides, G.M. Using magnetic levitataion for three dimensional self-assembly. submitted 
29. Whitesides, G.M. \& Boncheva, M. Beyond molecules: Self-assembly of mesoscopic and macroscopic components. Proc. Natl. Acad. Sci. 99, 4769-4774 (2002).

30. Olenyuk, B., Whiteford, J.A., Fechtenkötter, A. \& Stang, P.J. Self-assembly of nanoscale cuboctahedra by coordination chemistry. Nature 398, 796-799 (1999).

31. Cölfen, H. \& Mann, S. Higher-order organization by mesoscale self-assembly and transformation of hybrid nanostructures. Angew. Chem. Int. Ed. 42, 2350-2365 (2003).

32. Bowen, L. \& Radin, C. Densest Packing of Equal Spheres in Hyperbolic Space. Discrete Comput. Geom. 29, 23-39 (2003).

33. Scott, G.D. Packing of Spheres: Packing of Equal Spheres. Nature 188, 908-909 (1960).

34. Wu, H., Thalladi, V.R., Whitesides, S. \& Whitesides, G.M. Using Hierarchical SelfAssembly To Form Three-Dimensional Lattices of Spheres. J. Am. Chem. Soc. 124, 1449514502 (2002).

35. Meng, G., Arkus, N., Brenner, M.P. \& Manoharan, V.N. The Free-Energy Landscape of Clusters of Attractive Hard Spheres. Science 327, 560-563 (2010). 
Figure 1. a) Schematic of the device used for MagLev overlaid with a cross-section of the calculated magnetic field in the $y$-z plane centered on the magnets, and with a cross-section of the magnetic field in the $x-y$ plane between the magnets; b) Photographs of clusters of levitating PMMA spheres (5/32-in / 4-mm diameter) levitating in $1.3 \mathrm{M}$ aq. $\mathrm{MnCl}_{2}$. The left column shows examples of clusters that form as-placed in the device, and the right column shows the same cluster after mechanical agitation for two minutes. As-placed clusters form sheets that, for fewer than eight spheres, are generally, but not always close-packed (contrast the cluster with five spheres to the clusters of six and seven spheres). The sixth sphere in the agitated column lies above the plane of the other five and is shown shaded in the schematic.

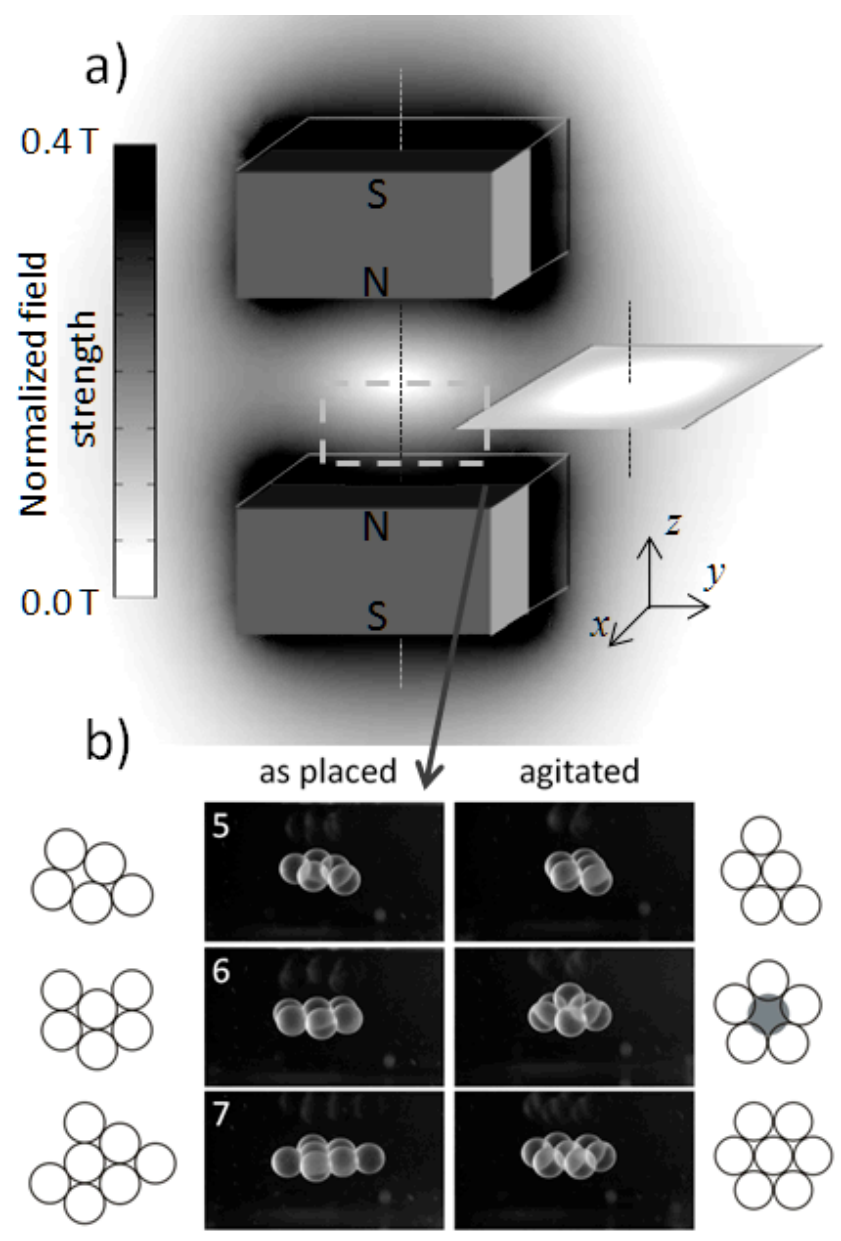


Figure 2: Photographs showing the progressive formation of a SA cluster as spheres are added from above. The center of mass of the crystal levitates at constant height. Magnet-to-magnet separation is denoted on the right. The shape of the clusters follows the shape of the magnetic trap, with the flattening of the cluster apparent as the distance between the magnets is increased (COMSOL calculations are shown on the left). Bottom row: the addition of a container (standard cuvette, $10 \mathrm{~mm}$ wall-to-wall distance) induces FCC packing in the clusters, in contrast with the less-ordered clusters induced by the magnetic template alone. Numbers given as column headings denote the number of spheres in the cluster. Arrows point to the center of mass of the levitating clusters.

\section{Figure 2}

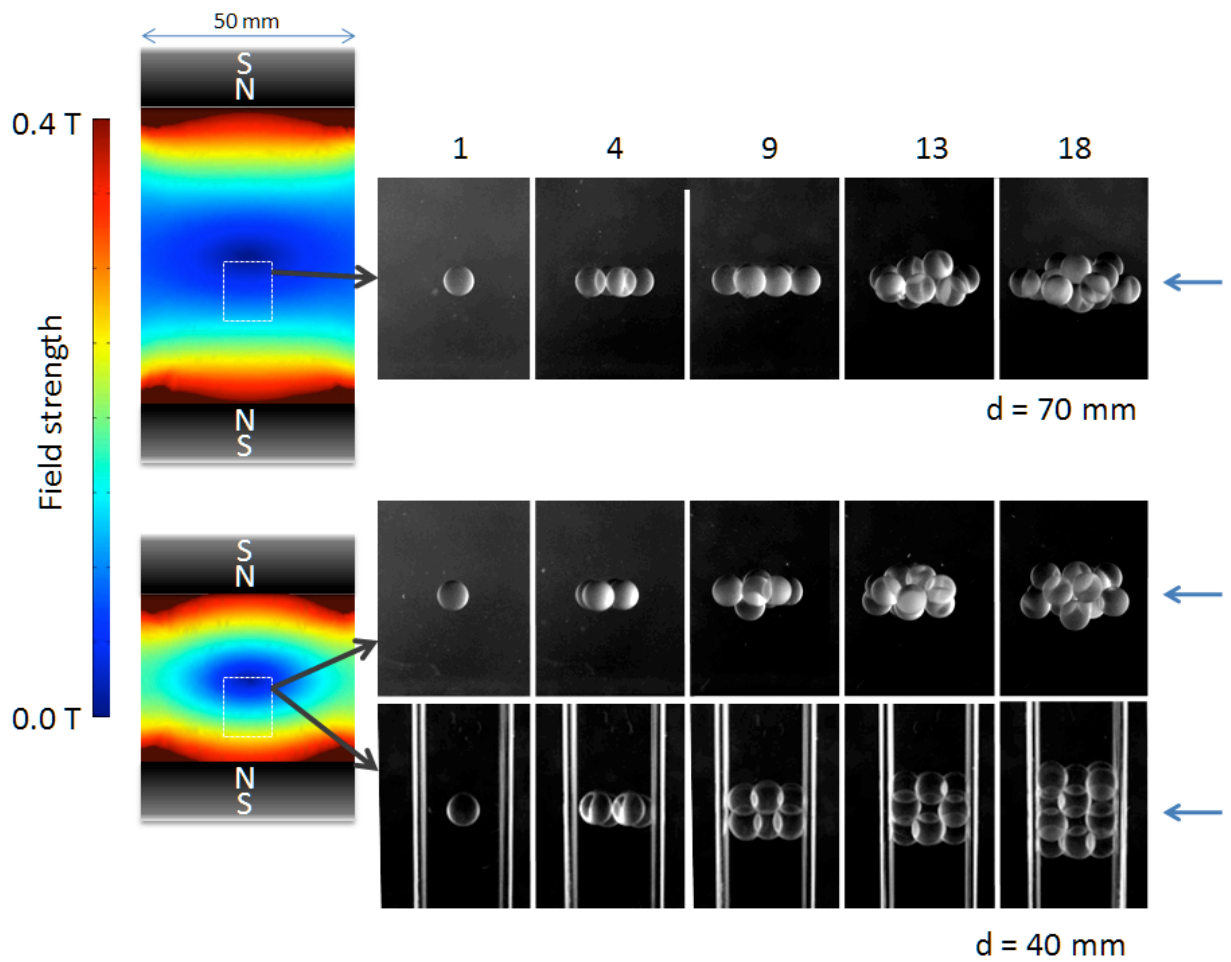


Figure 3. Pictures of levitating spheres ordered by co-levitating templates (a top view of the shape of the template, in gray, is shown schematically along with the placement of spheres). a) fcc ordering in the freely levitating cluster can be promoted by the addition of a flat, rigid colevitating template (PMMA disks in the image). Right, two disks were first designed and fabricated to levitate at different heights by applying polytetrafluoroethylene tape (bottom disk) and polyvinyl chloride tape (top disk); all components were placed in the container simultaneously and the container was placed in the magnetic field. Agitation produced the ordered cluster. The structures obtained were reproducible: the exact location of the spheres varied, but the packing and the general shape of the cluster always remained the same. b) Enveloping templates: laser-cut PMMA templates ordering 1/8-in / 3-mm diameter spheres. c) Displacing templates: the top image shows a 1/4-in / 6.4-mm diameter PMMA sphere templating the assembly of 1/16-in / 1.6-mm diameter spheres, the middle is a notched template that locks the position of twelve 5/32-in / 4-mm diameter spheres with respect to one another, and the bottom is the same template with four spheres. The scale bars are 1/4-in / $6.3 \mathrm{~mm}$ in all photographs. 
Figure 3
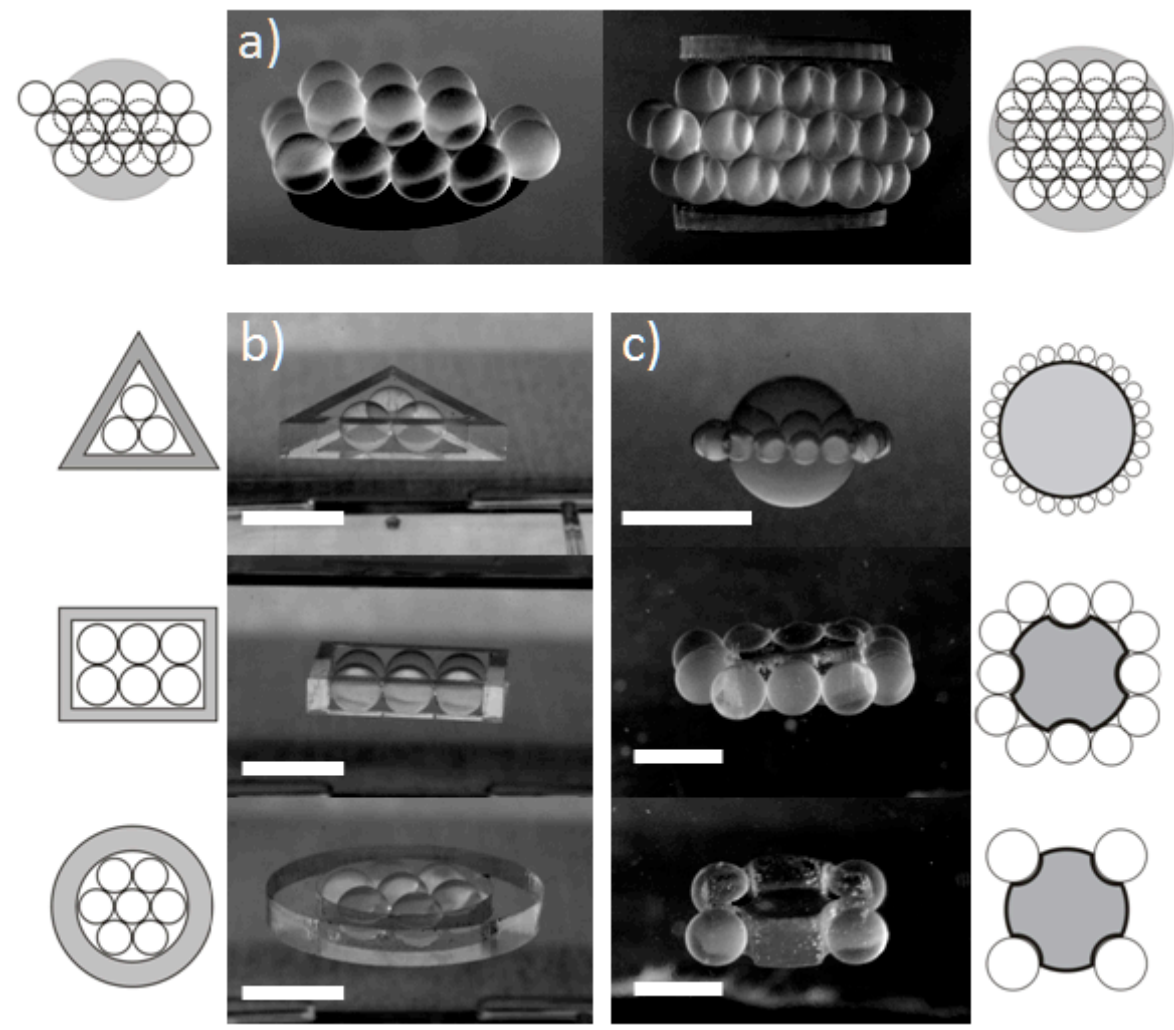
Figure 4. Photographs demonstrating the concept of density-based separation and self assembly of multiple objects. a) Non-templated spheres assemble into separate layers based on density: (from bottom to top) black is neoprene, gray is Torlon and lighter gray is polyvinylchloride, spheres are 1/4-in / $6.3 \mathrm{~mm}$ in diameter. b) A 1/4-in / 6.3-mm diameter Delrin sphere simultaneously templates 1/16-in 1.6-mm diameter Delrin spheres around its equator (white) and Torlon spheres (gray) below them, and 5/64-in / 2 mm-diameter rubber spheres (black) at its bottom pole. Scale bar is $1 / 4 " / 6.3 \mathrm{~mm}$.

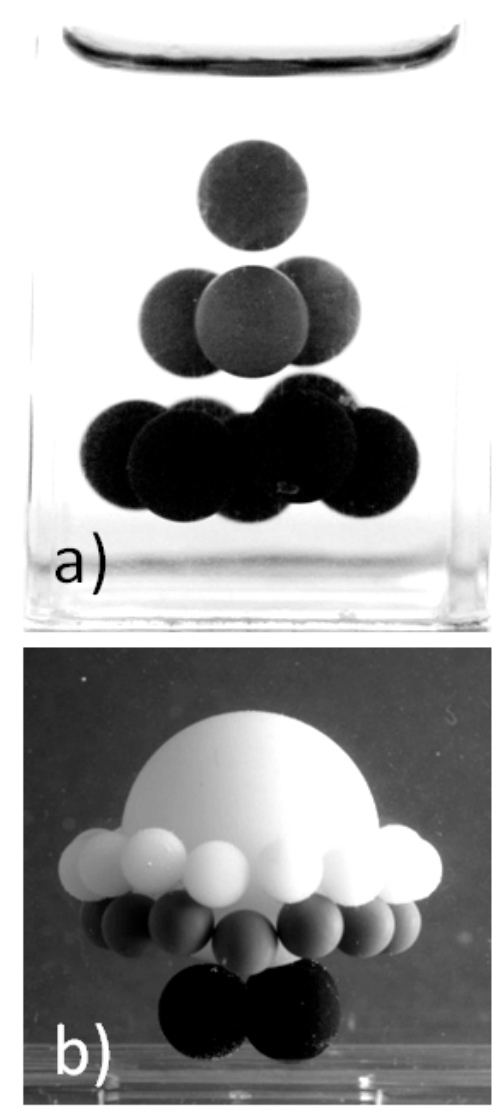


Figure 5 The self-assembly of complex objects can be programmed by patterning the density of the levitating objects. Here, interlocking pieces were prepared by gluing polymer spheres to prepared $13 \times 8 \mathrm{~mm}$ acrylic sheets (red). White layers are adhesive-backed Teflon. Each object is programmed to levitate at a different levitation height when placed in the same container together; after the objects self-aligned by placing the container in the magnetic field, the magnetto-magnet distance was reduced from $60 \mathrm{~mm}$ to $30 \mathrm{~mm}$ to bring them into contact. The different spheres are: $\mathrm{A}$ - acrylic (PMMA), $\mathrm{P}$ - polystyrene and $\mathrm{T}-$ Torlon. The magnets were $3.5 \times 2.5 \times$ 1 in $/ 89 \times 64 \times 25 \mathrm{~mm}$.

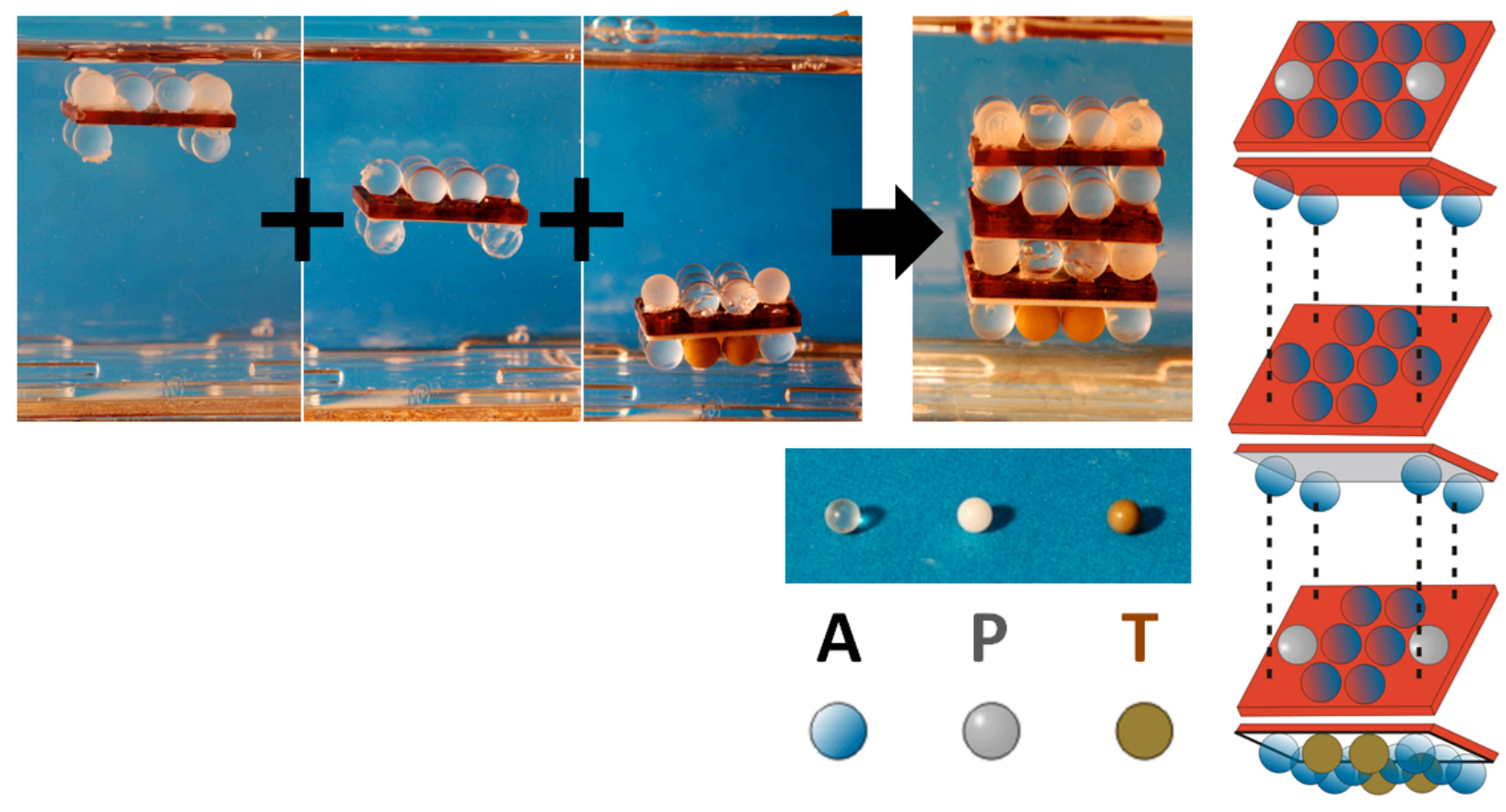


Figure 6: Self assembled clusters of spheres can be glued permanently by introducing an emulsion of optical adhesive to the paramagnetic solution, allowing for the adhesive to coat the surface and curing it under UV-light. The left figure shows a levitating cluster of seven 5/32-in / 4-mm diameter spheres; the cluster was subsequently glued and removed from the solution, shown center. Right is a SA cluster of 40 spheres that have been glued together. Scale-bars are $6.3 \mathrm{~mm}$.

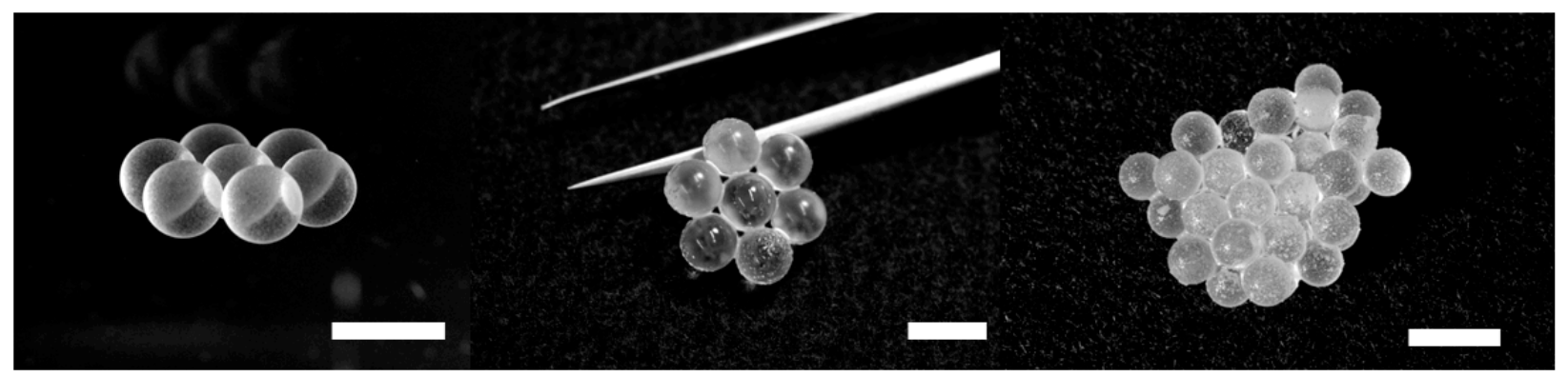

